\title{
Correction to: Year based EM-index: a new approach to evaluate the scientific impact of scholars
}

\author{
Anand Bihari ${ }^{1}$ (D) Sudhakar Tripathi ${ }^{1}$ \\ Published online: 26 July 2018 \\ (C) Akadémiai Kiadó, Budapest, Hungary 2018
}

\section{Correction to: Scientometrics (2018) 114:1175-1205 \\ https://doi.org/10.1007/s11192-017-2625-2}

In the original publication, Acknowledgements was published with incomplete information. The complete Acknowledgements is given in this correction.

Acknowledgements The authors wish to express their gratitude to anonymous reviewers for their valuable comments. The authors would like to acknowledge the Ministry of Electronics \& Information Technology (MeitY), Government of India, for providing financial assistance for this research work through "Visvesvaraya $\mathrm{PhD}$ Scheme for Electronics \& IT".

The original article can be found online at https://doi.org/10.1007/s11192-017-2625-2.

Anand Bihari

anand.cse15@nitp.ac.in; csanandk@gmail.com

Sudhakar Tripathi

stripathi.cse@nitp.ac.in; p.stripathi@gmail.com

1 Department of Computer Science and Engineering, National Institute of Technology Patna, Bihar, India 